\title{
Development of a Contingency Gas Analyzer for the Orion Crew Exploration Vehicle
}

\author{
Bill Niu ${ }^{1}$ and Kenneth Carney ${ }^{2}$ \\ Hamilton Sundstrand, Pomona, CA, 91737, USA \\ George Steiner ${ }^{3}$ \\ Hamilton Sundstrand, Pomona, CA, 91737, USA \\ William O'Hara ${ }^{4}$ \\ Lockheed-Martin, Houston, TX, 77058, USA \\ and \\ John Lewis ${ }^{5}$ \\ NASA, Houston, TX, 77058, USA
}

\begin{abstract}
NASA's experience with electrochemical sensors in a hand-held toxic gas monitor serves as a basis for the development of a fixed on-board instrument, the Contingency Gas Analyzer (CGA), for monitoring selected toxic combustion products as well as oxygen and carbon dioxide on the Orion Crew Exploration Vehicle (CEV).

Oxygen and carbon dioxide are major components of the cabin environment and accurate measurement of these compounds is critical to maintaining a safe working environment for the crew. Fire or thermal degradation events may produce harmful levels of toxic products, including carbon monoxide (CO), hydrogen cyanide (HCN), and hydrogen chloride $(\mathrm{HCl})$ in the environment. These three components, besides being toxic in their own right, can serve as surrogates for a panoply of hazardous combustion products. On orbit monitoring of these surrogates provides for crew health and safety by indicating the presence of toxic combustion products in the environment before, during and after combustion or thermal degradation events.

Issues identified in previous NASA experiences mandate hardening the instrument and components to endure the mechanical and operational stresses of the CEV environment while maintaining high analytical fidelity. Specific functional challenges involve protecting the sensors from various anticipated events- such as rapid pressure changes, low cabin pressures, and extreme vibration/shock exposures- and extending the sensor lifetime and calibration periods far beyond the current state of the art to avoid the need for on-orbit calibration.

This paper focuses on lessons learned from the earlier NASA hardware, current testing results, and engineering solutions to the identified problems. Of particular focus will be the means for protecting the sensors, addressing well known cross-sensitivity issues and the efficacy of a novel self monitoring mechanism for extending sensor calibration periods.
\end{abstract}

\section{Introduction}

\section{A. CGA OVERVIEW}

The Contingency Gas Analyzer (CGA) is an element of the Fire Detection Subsystem of the Environmental Control and Life Support System (ECLSS) for the Orion Crew Exploration Vehicle (CEV). During the CEV mission, CGA continuously monitors the cabin atmosphere for oxygen $\left(\mathrm{O}_{2}\right)$, carbon dioxide $\left(\mathrm{CO}_{2}\right)$, carbon monoxide $(\mathrm{CO})$, hydrogen cyanide $(\mathrm{HCN})$ and hydrogen chloride $(\mathrm{HCl})$ and total cabin pressure. The role of CGA has evolved from its original purpose of monitoring combustion gases during and after a fire or thermal degradation

\footnotetext{
${ }^{1}$ Corporate Fellow, HS-Pomona, 2771 N. Garey Ave., Pomona, CA, 91737, AIAA Member Grade.

${ }^{2}$ Instrument Scientist, HS-Pomona, 2771 N. Garey Ave., Pomona, CA, 91737, AIAA Member Grade.

${ }^{3}$ Program Manager, HS-Pomona, 2771 N. Garey Ave., Pomona, CA, 91737, AIAA Member Grade..

${ }^{4}$ Principle Engineer, Department Name, Address/Mail Stop, AIAA Member Grade.

${ }^{5}$ Insert Job Title, Department Name, Address/Mail Stop, AIAA Member Grade.
} 
event. In addition to monitoring toxic combustion products, the CGA also serves as the primary cabin pressure control sensor, as a backup/emergency monitor for carbon dioxide and oxygen, and as a complement to the cabin's smoke/particulate based fire detector.

The CGA is mounted on the forward bulkhead of the crew cabin to access cabin air. CGA's primary I/O interface is through the ECLS software on the vehicle's master computer (VMC). Under certain contingencies, including post-landing, when that interface is not available the CGA includes a remote display and alarm unit to apprise the crew of the cabin air composition and related potential hazards.

In addition to their direct toxicity $\mathrm{CO}, \mathrm{HCN}$ and $\mathrm{HCl}$ serve as surrogates to indicate the presence or absence a panoply of potentially toxic combustion products. In a post-fire cleanup, if these constituents are successfully removed, then one may infer that the cabin atmosphere has been rendered safe for the crew. Oxygen and $\mathrm{CO}_{2}$ concentrations on the other hand are ongoing indicators of a well-controlled life support atmosphere. The oxygen concentration must be balanced between a level that is high enough to support crew health yet below a level that presents a flammability hazard and $\mathrm{CO}_{2}$ must be kept below a maximum threshold to ensure crew comfort, performance and safety. The Contingency Gas Monitor is a key component for monitoring these gases at various stages of the Orion mission.

\section{B. ELECTROCHEMICAL SENSORS}

The CGA uses a suite of 5 electrochemical (EC) sensors for detecting $\mathrm{O}_{2}, \mathrm{CO}, \mathrm{HCN}$ and $\mathrm{HCl}$. Carbon dioxide is monitored by non-dispersive infrared (NDIR) spectrometry. The use of electrochemical sensors offers significant benefits with respect to power, weight and size when compared with other mature detection technologies. Applying electrochemical sensor technology in a space environment presents far greater technical challenges than the spectroscopic detection of carbon dioxide and, thus, issues related to the EC sensors are the principle subject of this paper. Electrochemical sensors have a long history and wide use in industrial and chemical hygiene settings and have previously flown in NASA's hand-held "Compound Specific Analyzer" used on Shuttle and ISS missions. ${ }^{1}$

All of the electrochemical sensors in CGA detect target analytes by oxidizing (or reducing, in the case of oxygen) analyte molecules at one electrode of an electrochemical cell. The diagram in Fig. 1 illustrates the construction and operation of a sensor. As a sample gas passes across the face of the sensor, analyte begins to diffuse through the diffusion barrier and a steady state concentration gradient is established within a few seconds. The concentration of analyte within the cell is kept at zero in the cell as the electrochemical reaction rapidly consumes any analyte molecules passing through the barrier. In this steady state, analyte from the sample gas passes through the

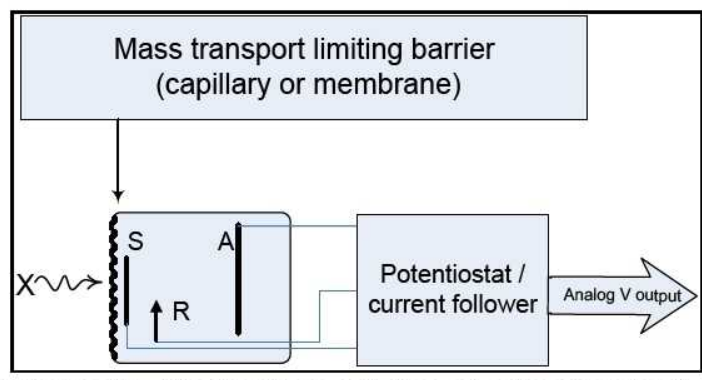

Figure 1. Schematic illustration of the electrochemical gas sensing. Analyte $(X)$ passes through the diffusion barrier, in proportion to the gas phase concentration, under the influence of the concentration gradient created by the consumption of $X$ at the Sensing electrode (S). The analog circuit maintains the potential of the sensing electrode relative to a reference electrode $(R)$ and measures the current generated during the electrochemical reaction. Many sensors use an auxiliary electrode (A) to improve control of the potential difference between $S$ and $R$.

diffusion barrier and into the cell in proportion to the gas phase concentration (or partial pressure) according to Fick's laws. It is the diffusion limited mass transport, not the electrochemistry, that imparts the quantitative relationship between sensor response and concentration. Selective oxidation or reduction of analyte at the electrode provides selectivity toward specific target analytes. The selectivity of the different sensors is effected through the use of various combinations of catalytic electrode material and electrode potentials. Analog electronics circuits fix the potential of the sensing electrode relative to a reference and measures the number of analyte molecules passing through the barrier via the current generated during the electrochemical reaction. All CGA electrochemical sensors except the oxygen sensor use potentiostatic control to fix the electrode potentials at specific values. The oxygen sensor, however, is a galvanic cell in which the electrode potential is poised by the thermodynamics of the cell and current flows due to the spontaneous reduction of oxygen at the sensing electrode. The diffusion barriers for all of the electrochemical gas sensors used in CGA, except for oxygen, use permeable membrane diffusion barriers. The oxygen sensor uses a small capillary orifice as the diffusion barrier. 


\section{IMPLEMENTATION}

Two particular problems observed for EC sensors in general in previous spaceflight applications are pressure induced physical damage and leakage and calibration changes over time. Overcoming the second of these- frequent recalibration- promised to be a significant challenge as accommodating integration schedules and pre-launch operations extended the sensor functionality timeframe significantly beyond the current state of the art. In addition, sensor-specific issues of concern for CEV were the expected cross-sensitivity of $\mathrm{CO}$ sensors toward hydrogen, drifting of $\mathrm{O}_{2}$ sensor response, and long response and recovery times for $\mathrm{HCN}$ sensors. Finally, a minor concern was maintaining adequate airflow across the sensor surface. As most commonly used, EC gas sensors are "passive" sensors in that they rely on ambient air circulation to move air across the surface of the diffusion barrier at a minimum velocity in order to maintain quantitative fidelity. The specific minimum velocity required is not well defined and ordinarily normal air circulation should meet this requirement. In still environments, however, the socalled face velocity may drop below the minimum, producing a stagnant layer adjacent to the diffusion barrier and a negative bias in the results.

\section{A. PRESSURE TOLERANCE}

An initial concern in using EC sensors for CGA was whether they could physically survive anticipated depressurization/repressurization events. Previous NASA experience indicated that EC sensors were susceptible to damage, ranging from pressure induced response failure to actual leakage of electrolyte from the sensor body, when exposed to pressure cycling. One of the driving scenarios for CGA's initial role involves cabin depressurization and several other Orion activities involve potential cabin depressurization. Thus, it was mandatory that the CGA sensors survive relatively rapid depressurization and repressurization events. The sensors selected for use in CGA use zero headspace cells with stabilized liquid electrolytes to minimize the likelihood of expanding headspace gases forcing electrolyte through the diffusion barrier, the only potential electrolyte pathway to the environment. CGA's primary safeguard against both low pressures and extreme rates of pressure change is the incorporation of the sensors into a flow cell that is protected by two solenoid valves. The valves are controlled by software and are closed to isolate the sensors from the ambient environment when the pressure drops below the minimum operating pressure or when the cabin pressure is changing at a rate deemed to be harmful to the sensors. During operation, a small sample pump draws ambient air through the flow cells, which are designed to enhance mixing of the sample and to inhibit the development of a stagnant air layer adjacent to the diffusion barrier during operation. Thus the CGA can produce accurate results even in very still environments, so long as the air at the sample inlet is representative of the cabin atmosphere.

The pressure thresholds for closing the valves are still being refined, but pressure testing to date has indicated that the sensors are not particularly susceptible to damage by short exposure to pressures as low as 5 psia or by repeated pressure cycling at rates as high as \pm 4 psi/minute between 5 and 15 psia. Barometric pumping of liquid electrolyte from the sensors has not been observed in any pressure tests to date. After exposure to pressures as low as $5 \mathrm{psi}$ for several hours or more, all of the sensors typically recovered and functioned properly at normal operating pressures. The low partial pressure of water, even at high relative humidity, in low pressure environments caused some sensor types to dry out when exposed to longer periods at low total pressures. For example, a series of tests in which sensor response was evaluated following exposures to very low pressures $(<2$ psia $)$ showed $\mathrm{HCN}$ sensors to be significantly affected by extended exposure to very low pressures while $\mathrm{O}_{2}$ sensors were unaffected. A sample of $\mathrm{O}_{2}$ sensors showed no adverse effects after more than 300 cumulative hours at pressures below 2 psia. A sample of HCN sensors, on the other hand, showed $10 \%$ sensitivity loss after less than 24 hours at 2 psia and complete failure after less than 140 cumulative hours at 2psia. Post-failure inspection of one $\mathrm{HCN}$ sensor determined that the sensor was completely desiccated; but it showed no indication of electrolyte leakage, suggesting that evaporative loss of water from the electrolyte was responsible for the failure. Further, because their physical construction is so similar, the $\mathrm{HCN}$ sensor should reliably model the effects of low pressures on the $\mathrm{HCl}, \mathrm{CO}$, and $\mathrm{H}_{2}$ sensors.

\section{B. ANALYTICAL ROBUSTNESS}

The second major concern with using COTS electrochemical sensor technology for the CEV application was sensor longevity and calibration durability. Currently quoted sensor lifetime estimates for COTS sensors generally are near the 2 year CEV requirement, but commercial vendors typically support these lifetime targets through liberal sensor replacement policies. This replacement approach works well in most commercial settings, but is inadequate when sensor replacement is not feasible and high reliability is required. The statistics surrounding COTS lifetime estimates are vague and the probability of getting a sensor that doesn't meet the stated lifetime for one reason or another is not negligible. Furthermore, current sensor lifetime estimates anticipate generally decreasing sensor 
sensitivity over the sensor life, necessitating frequent recalibration. COTS sensors typically must be recalibrated every 1 to 3 months, particularly if high analytical accuracy is desired. Frequent recalibration is not a realistic option for the CEV application, especially during the active mission phase and particularly for highly toxic gases such as $\mathrm{HCl}$ and $\mathrm{HCN}$. While it is commonly expected that sensor sensitivity will decrease with sensor age, the extent of that decrease as the sensor ages is virtually uncharacterized for general production sensors.

The problem of sensor aging and calibration drift is addressed in CGA by the implementation of enhanced acceptance criteria when the sensors are initially purchased and by the use of complementary built-in state-of-health tests performed intermittently during CGA operation. In addition to working with the vendor through conventional materials control approaches to quality, CGA is implementing an extended criteria set for sensor acceptability. Sensors are screened based on sensitivity and background signal (an indirect indicator of purity), but they are further evaluated through a series of tests to verify that the chemical composition and physical characteristics (e.g., electrode spacing, surface area) of the underlying electrochemical cells is as consistent as possible. Expanding the tests to encompass certain physical traits is important as current manufacturing processes allow for significant, poorly controlled, variations between production batches, especially over periods of time that may encompass changes in materials suppliers and production personnel. Expanding the number of selection criteria, i.e., the number of factors that must match to put a sensor in the accepted population increases the likelihood that sensors not only will behave similarly with respect to their initial analytical performance, but more importantly that they will behave similarly with respect to how analytical performance changes as the sensors age. By more tightly defining the population of sensors from the beginning, one can improve the statistical reliability of subsequent built-in test results for tracking sensor/calibration aging. Once the population of chosen sensors is selected, CGA uses a sub-sample of that population to define the age-response characteristic for the population. Subsequently at various times during normal operation, the CGA software executes the built-in test, estimates the current sensor accuracy and, if necessary, makes adjustments to the sensor calibration based on the test results. A small set of sensors was subjected to accelerated aging by exposure to various elevated temperatures (up to $40^{\circ} \mathrm{C}$ ) and dry environments (down to $12 \% \mathrm{RH})$. The results showed significant improvement in calibration stability using the built-in testing procedure with a primitive sensitivity drift model. Fig. 2 shows some results from the accelerated aging study with HCN sensors. Currently additional validation of the approach is underway using a larger sample of sensors and long-term sensor lifetime studies.

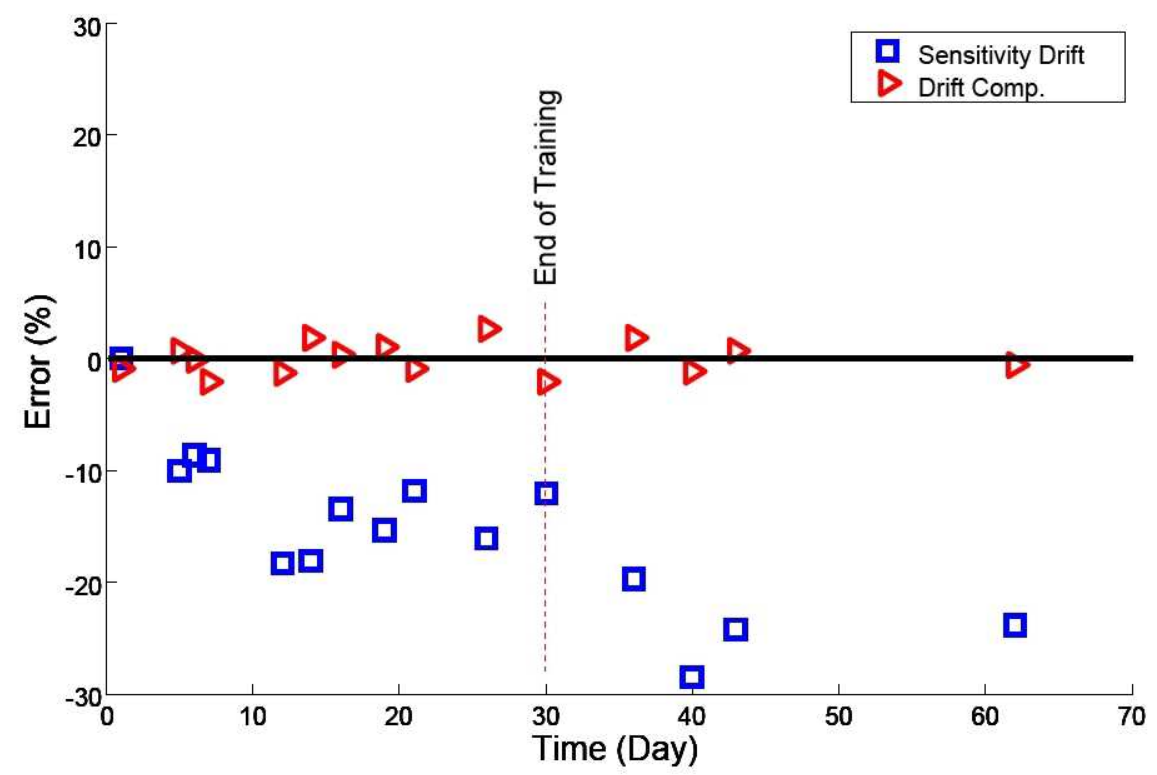

Figure 2. Sensitivity Drift Compensation. HCN sensor sensitivity drift was reduced to less than $3 \%$ by applying a primitive drift compensation model during a 2 month accelerated aging test. Data prior to Day 29 were used to train the model.

Drift in oxygen sensor response has been reported as an issue in previous spaceflight applications ${ }^{2}$ and was a consideration for CGA from the inception of its $\mathrm{O}_{2}$ monitoring role. A set of oxygen sensors is undergoing lifetime drift testing. In testing to date, the only sensors that have shown significant drift are sensors that are well into their expected lifetime. Galvanic $\mathrm{O}_{2}$ sensors function much like a battery and appear, like many other types of batteries, to 
hold a steady output for an extended period of time followed by a relatively brief period of rapidly decreasing output during their final stages of discharge. Consequently sensor age is one of the primary acceptance criteria for CGA $\mathrm{O}_{2}$ to ensure the maximum use time with minimal drift. In addition, techniques for minimizing sensor exposure to $\mathrm{O}_{2}$ during non-operational periods are being explored. The oxygen sensors are included in the enhanced acceptance testing regime described above and a database of acceptance testing results is being created to correlate observed sensor lifetime performance with initial chemical-physical characteristics. One purpose of the database is to identify latent characteristics that can predict sensors that will prematurely enter their "high drift" phase.

\section{CARBON MONOXIDE-HYDROGEN CROSS SENSITIVITY}

Carbon monoxide is arguably the most important of the three "combustion gases" monitored by CGA. It is the most persistent of the three and is generally present in higher concentrations, so it provides the most conservative surrogate for other, unmonitored, hazardous combustion products. In addition, CO provides a complement to Orion's smoke/particulate based fire detector because there are few, if any, $\mathrm{CO}$ sources in the cabin other than combustion. In order to serve effectively as a fire indicator, the $\mathrm{CO}$ level must be reliably monitored at low ppmv levels. The cross sensitivity of electrochemical CO sensors to hydrogen is well known ${ }^{3}$, so the presence of significant hydrogen concentration in the cabin poses a problem for precise monitoring of $\mathrm{CO}$ for fire detection. Estimates of hydrogen sources, primarily metabolic, in the cabin indicate that concentrations could exceed 150ppmv and may be quite variable. Further, some $\mathrm{CO}$ sensors showed not only a cross-sensitivity toward hydrogen, but also negative interference by hydrogen when both $\mathrm{CO}$ and $\mathrm{H}_{2}$ were present. In other words, hydrogen produces a positive sensor response in air samples containing only $\mathrm{H}_{2}$, but in samples containing both $\mathrm{CO}$ and $\mathrm{H}_{2}$, it also reduces the sensor response to $\mathrm{CO}$. In Table I sensors 2 and 3 exhibit a negative interference due to hydrogen, but sensor 1 does not. Selectivity, $\mathrm{K}$, is a measure of cross-sensitivity and is calculated as the ratio of the sensor's sensitivity toward $\mathrm{CO}$ in air to its sensitivity toward $\mathrm{H}_{2}$ in air. Selectivity alone is not an adequate measure of sensor suitability for the CEV application.

\begin{tabular}{|c|c|c|c|}
\hline & Sensitivity (1 & $\mathrm{pmv} \mathrm{CO})^{1}$ & \\
\hline $\begin{array}{c}\text { H2 conc'n } \\
\text { (ppmv) }\end{array}$ & $\begin{array}{c}\text { Sensor 1 } \\
(\mathrm{K}=2)^{2}\end{array}$ & $\begin{array}{c}\text { Sensor } 2 \\
(\mathrm{~K}=4)\end{array}$ & $\begin{array}{c}\text { Sensor 3 } \\
(\mathrm{K}=7)\end{array}$ \\
\hline 0 & 1.77 & 1.60 & 1.56 \\
\hline 40 & 1.83 & 1.29 & 1.31 \\
\hline 80 & 1.73 & 0.93 & 0.66 \\
\hline
\end{tabular}

For CGA, the $\mathrm{CO}$ sensor is obtained from a manufacturer that produces stable sensors having selectivities between 20 and 30 while at the same time showing no significant negative interference by hydrogen at concentrations up to $200 \mathrm{ppmv} \mathrm{H}_{2}$. This sensor is paired with a second sensor that is sensitive to both $\mathrm{CO}$ and $\mathrm{H}_{2}$, but with a much lower selectivity toward $\mathrm{CO}$. Neither of the two sensors is completely selective toward either $\mathrm{CO}_{\text {or }} \mathrm{H}_{2}$, but the selectivity difference is large and stable enough that $\mathrm{CO}$ and $\mathrm{H}_{2}$ concentrations can be reliably calculated from a simple linear equation. Fig. 3 shows an early test using a simple algorithm to correct the sensor response in the presence and absence of hydrogen background. 


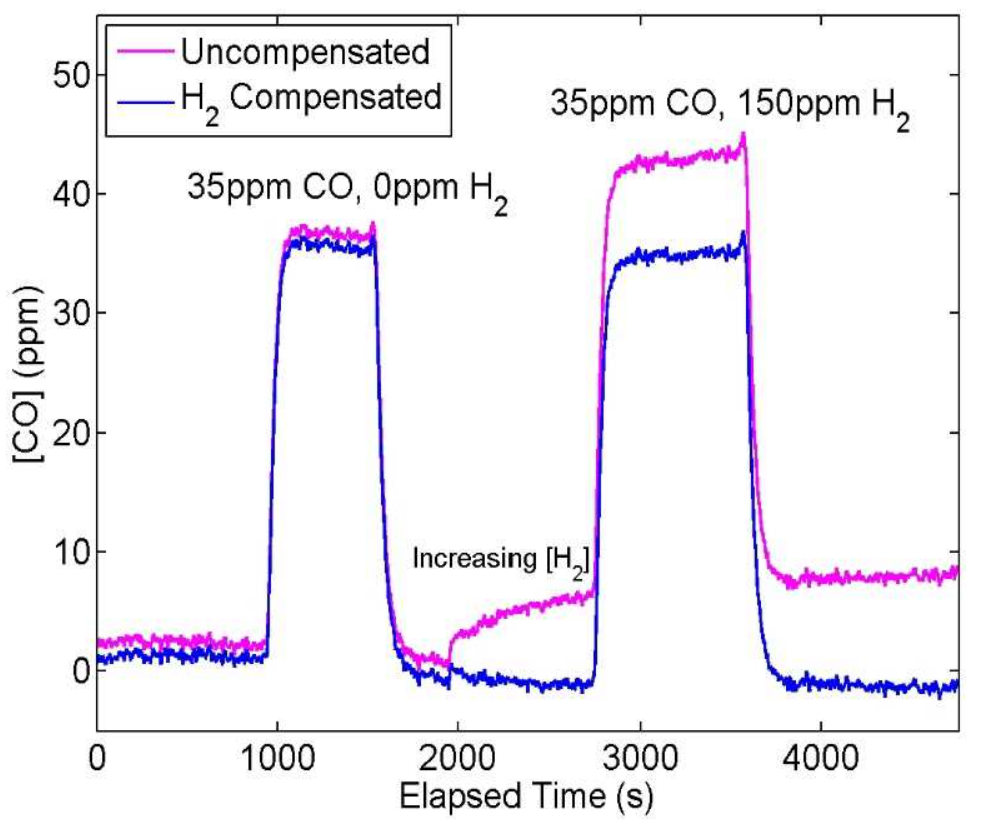

Figure 3. Cross Sensitivity Compensation. Carbon monoxide detection was compensated for hydrogen interference using a two sensor compensation technique. The uncompensated output for a $\mathrm{CO}$ sensor with a selectivity of approximately 25 showed positive bias greater than 5 ppmv $\mathrm{CO}$ in the presence of $150 \mathrm{ppmv} \mathrm{H}_{2}$. Compensation with a simple linear correction of the $\mathrm{CO}$ concentration based on a second sensor with much lower selectivity reduced the error to less than 1ppmv CO. Neither sensor was completely specific for either $\mathrm{CO}$ or $\mathrm{H}_{2}$ but their selectivities were different and consistent.

\section{SENSOR RESPONSE TIME}

CGA electrochemical sensor response times, measured as the time required to reach $90 \%$ of the steady state value $\left(t_{90}\right)$, range from 20 seconds for $\mathrm{O}_{2}$ to 30 seconds for $\mathrm{CO}$ and $\mathrm{H}_{2}$, to slightly less than 60 seconds for $\mathrm{HCl}$. These speeds fall within the requirements for the CEV application. The response time for $\mathrm{HCN}$, however, is more problematic, with $\mathrm{t}_{90}$ typically $300-400$ seconds. Furthermore, the response time for HCN sensors varies widely between sensors and it is not uncommon to find sensors with $t_{90}$ greater than 600 seconds. Finally, the response time of HCN sensors increases greatly toward the end of the sensor's useful life; it is not lack of sensitivity per se that defines the end of life for these sensors, but rather an increasingly sluggish response. These behaviors appear to be typical of commercial HCN sensors and required additional consideration in CGA development as the response delay could lead to underestimating $\mathrm{HCN}$ concentration when $\mathrm{HCN}$ is increasing and overestimating concentration when $\mathrm{HCN}$ is decreasing. The latter condition is more germane to the CEV application in that CGA's HCN detection function is intended specifically for establishing a safe environment during cleanup following event that may have generated hazardous levels of $\mathrm{HCN}$ and not for detecting the onset of HCN contamination. In this application the relevant risk is not endangerment due to under-reporting the $\mathrm{HCN}$ concentration, but rather wasting valuable time and resources as the reported concentration lags behind a decreasing $\mathrm{HCN}$ concentration. Thus, the response time for $\mathrm{HCN}$ is not as critical as for $\mathrm{O}_{2}$ or $\mathrm{CO}$, two components for which both increasing and decreasing trends are important to crew health and safety. Careful selection of $\mathrm{HCN}$ sensors can provide CGA with a marginally reasonable response time between 5 and 6 minutes for HCN, but the tendency of the response time to increase with age must be checked. In the accelerated aging tests mentioned above one of the HCN sensors under test showed a sudden increase in response time, to the point of failure. The built-in test being used to monitor sensor aging showed a marked deviation prior the failure and appears to be an effective means for anticipating this type of sensor failure. Efforts to characterize the onset of excessively slow response in an otherwise acceptable sensor and to identify contributing factors are currently underway as part of sensor lifetime testing.

\section{SUMMARY}

Electrochemical sensors provide significant cost, power and weight advantages for use in a contingency gas analyzer for the Orion Crew Exploration Vehicle. Solutions to issues identified in prior in-flight use of electrochemical sensors and to issues unique to the CEV mission have been in development as part of the Hamilton Sundstrand CGA effort. A flow cell approach to delivering sample to the sensors provides both a means to protect the sensors from pressure extremes and an insensitivity to 
ambient air movement that could otherwise cause quantitative errors. A two sensor approach to monitoring CO provides a robust and simple means for making accurate, low-level $\mathrm{CO}$ measurements in the presence of much higher, and possibly fluctuating, concentrations of interfering hydrogen. The CEV application requires calibration stability far beyond the state of the art for commercial sensors. This challenge is being addressed by the use of a stringent acceptance protocol and a complementary built-in test program that can track sensor changes in-situ during normal operation. This approach has been demonstrated in accelerated aging tests and was able both to compensate for sensor calibration drift and to predict impending failure of a sensor. Additional lifetime testing is currently underway to refine and validate these approaches.

\section{ACKNOWLEDGEMENTS}

This work was performed under NASA Contract \#NNJ06TA25C

\section{REFERENCES}

${ }^{1}$ Limero, T., Beck, S., and James J.T., "The Portable Monitor for Measuring Combustion Products Aboard the International Space Station," SAE Technical Paper Series 2002-01-2298, 32nd International International Conference on Environmental Systems, San Antonio, Texas, July 2002.

${ }^{2}$ Limero, T., Beck, S., and James J.T., "Development and Performance of the Oxygen Sensor in the CSA-CP Aboard the International Space Station," SAE Technical Paper Series 2004-01-2337, $34^{\text {th }}$ International Conference on Environmental Systems, Colorado Springs, Colorado, July 2004.

${ }^{3}$ Champagne, D. McCarrick, A. Rose-Pehrsson, S.L., Evans, T.W., Werner, J., "Use of Portable Gas Analyzers in an Environment with High Background Hydrogen Levels," SAE Technical Paper Series 2004-01-2338, 34 ${ }^{\text {th }}$ International Conference on Environmental Systems, Colorado Springs, Colorado, July 2004. 